\title{
Article
}

\section{Minimising the use of physical restraint in acute mental health services: The outcome of a restraint reduction programme ('RESTRAIN YOURSELF)}

Downe, Soo, Mckeown, Michael, Thomson, Gillian, Price, Owen, Whittington, Richard, Scholes, Amy, Duxbury, Joy, Jones, Fiona, Baker, John, Greenwood, Paul and Thygesen, Helena

Available at http://clok.uclan.ac.uk/27965/

Downe, Soo ORCID: 0000-0003-2848-2550, Mckeown, Michael ORCID: 00000003-0235-1923, Thomson, Gillian ORCID: 0000-0003-3392-8182, Price, Owen, Whittington, Richard, Scholes, Amy, Duxbury, Joy ORCID: 0000-00021772-6874, Jones, Fiona, Baker, John et al (2019) Minimising the use of physical restraint in acute mental health services: The outcome of a restraint reduction programme ('RESTRAIN YOURSELF). International Journal of Nursing Studies, 95 . pp. 40-48. ISSN 0020-7489

It is advisable to refer to the publisher's version if you intend to cite from the work. http://dx.doi.org/10.1016/j.ijnurstu.2019.03.016

For more information about UCLan's research in this area go to

http://www.uclan.ac.uk/researchgroups/ and search for <name of research Group>.

For information about Research generally at UCLan please go to http://www.uclan.ac.uk/research/

All outputs in CLoK are protected by Intellectual Property Rights law, including Copyright law. Copyright, IPR and Moral Rights for the works on this site are retained by the individual authors and/or other copyright owners. Terms and conditions for use of this material are defined in the policies page. 


\title{
Original Article
}

\section{Minimising the use of physical restraint in acute mental health services: The outcome of a restraint reduction programme ('REsTRAIN YOURSELF)}

\author{
ABSTRACT \\ Keywords \\ Physical restraint, coercion, mental health, intervention study

\section{Background}

Physical restraint is a coercive intervention used to prevent individuals from harming themselves or others. However, serious adverse effects have been reported. Minimising the use of restraint requires a multimodal approach to target both organisational and individual factors. The 'Six Core Strategies' developed in America, underpinned by prevention and trauma informed principles, is one such approach.

\section{Objective}

An adapted version of the Six Core Strategies was developed and its impact upon physical restraint usage in mental health Trusts in the United Kingdom evaluated. This became known as 'REsTRAIN YOURSELF. The hypothesis was that restraint would be reduced by $40 \%$ on the implementation wards over a six-month period.

\section{Design}

A non-randomised controlled trial design was employed.

\section{Setting}

Fourteen, adult, mental health wards from seven mental health hospitals in the North West of England took part in the study. Two acute care wards were targeted from all eligible acute wards within each site in negotiation with each Trust. The intervention wards (total $n=144$ beds, mean $=20.1$ beds per ward) and control wards (total $n=147$ beds, mean $=21.0$ beds per ward) were primarily mixed gender but included single sex wards also ( 2 female-only and 1 male-only in each group). All wards offered pharmacological and psychosocial interventions over short admission durations (circa 15 days) for patients with a mixture of enduring mental health problems.

\section{Method}

As part of a pre and post-test method, physical restraint figures were collected using prospective, routine hospital records before and 6 months after the intervention. Restraint rates on seven wards receiving the REsTRAIN YOURSELF intervention were compared with those on seven control wards over three study phases (baseline, implementation and adoption).

\section{Results}

In total, 1680 restraint incidents were logged over the study period. The restraint rate was significantly lower on the intervention wards in the adoption phase (6.62 events/1000 beddays, $95 \% \mathrm{Cl} 5.53-7.72)$ compared to the baseline phase $(9.38,95 \% \mathrm{Cl} 8.19-10.55)$. Across all implementation wards there was an average reduction of restraint by $22 \%$, with some wards showing a reduction of $60 \%$ and others less so (8\%). The association between ward type and study phase was statistically significant. 
Conclusion

In conclusion, it is possible that reductions in the use of physical restraint are achievable using a model such as the Six Core Strategies. This approach can be adapted for global settings and changes can be sustained over time with continued support.

\section{Acknowledgements}

This study was funded by the Health Foundation as part of the 'Closing the Gap' funding stream 2014-2016

Thank you to all the wards and participants who took part and gave their time so freely.

\section{INTRODUCTION}

Physical restraint is a coercive intervention commonly used to prevent individuals in mental health services from harming themselves or others (National linstitiute of Clinical Excellence 2015). Whilst policy and legislation specify that it should only be used as a 'last resort', it continues to be used routinely in mental health services in the UK and beyond (MIND 2013, Agenda 2017). A wide range of adverse effects have been reported as a result of the use of restraint ranging from patient and staff discomfort to injuries resulting in death (Duxbury et al 2011, Mohr et al 2003, Soininen et al. 2016). There is a growing recognition of the traumatic origins of mental distress and the potential for coercive practices to traumatise or retraumatise individuals (Sweeney et al. 2016, Bonner et al 2002). Additionally when surveyed, many practitioners feel unrest with the use of restraint particularly when trying to balance patient safety with patients' rights and less invasive procedures (Duxbury \& Whittington 2005, Duxbury 2015). There is a clear dissonance between wanting to manitain the safety of all in conflict situations whilst trying to ensure that patients are not retraumatised by approaches such as restraint and the resultant difficulties in maintainng a therapeutic relationship in such circumstances (Stewart et al 2009, Cleary et al 2012a). Staff have also reported injuries resulting in physical and psychological strain, stress, lack of confidence, prolonged sickness and disonnance (LeBel 2011). The cost to the organisations when staff are injured, under threat or stressed as a result of threatening behaviour is significant and impacts upon staff turnover, burnout and litigation (LeBel \& Goldstein 2005, Sanders 2009, Lebel et al 2014).

Minimising the use of physical restraint and other coercive measures such as seclusion and chemical restraint and improving patient safety, means considering how the current system works and what changes are needed to reduce harm or injury to the service user (Bowers et 
al 2015). Empirical evidence from Europe and North America clearly demonstrates that variations in restraint and seclusion rates are largely influenced by environmental, or contextual factors (Huckshorn 2004, Luciano et al. 2014, Riahi et al 2016, Cowman et al 2017) and that similar factors influence patients' subjective experiences of restrictive practices (Aguilera-Serrano et al. 2018). Unclear policies and guidelines, overcrowding, poor ward design, low or inflexible staff numbers, inexperienced staff, poor staff retention, poor information sharing and service user acuity have all been implicated (Duxbury \& Whittington 2005, National linstitiute of Clinical Excellence 2015). Various staff characteristics are also linked to aggression in mental health services, including negative interactional styles, provocative, authoritarian behaviour and poor communication skills (Bonner et al, 2002; Duxbury 2002; Glover, 2005; Tunde-Ayinmode \& Little, 2004; Dark et al 2012: Bowers 2014). Hence, a substantial body of evidence indicates that many seclusion and restraint episodes may be preventable if these contextual factors are addressed (Sanders 2009, Riahi et al 2016).

\section{BACKGROUND}

Events that threaten patient and staff safety such as violence, aggression and self-harm are not uncommon in mental health inpatient settings (Bowers et al 2015). Staff responses to these incidents frequently involve the use of practices which contain or restrict an individual and can cause serious physical harm (Paterson et al 2003) and adverse psychological effects (Bonner et al 2002, Rose et al 2015)

Restrictive interventions such as restraint are used globally (Cowman et al 2017, Bowers et al 2005, 2007, Steinert et al 2010) They include a specific range of practices such as physical restraint, seclusion and rapid tranquilisation. They can however, be much wider reaching in the use of locked wards, rigid cultures, environmental restrictions and many other oppressive approaches commonly referred to as coercive practices (Duxbury \& Whittington 2005, Cowman et al 2017). Rapid tranquilisation to treat and manage agitation and violence has also been recognized as a form of chemical restraint, which can be classed as a restrictive intervention (Department of Health 2014).

Cultural differences however, mean that across countries and other European services some forms of restrictive practices are more acceptable than others. For example, mechanical restraint is a feature of American, Canadian, Australian and some European approaches in acute services (Guzman-Parra et al 2016, Borckardt et al. 2011, Putkonen et al 2013) but its use is rarely allowed in mainstream acute settings in the UK (National Collaborating Centre for Mental Health 2015). Restrictive interventions can also have detrimental effects on 
therapeutic relationships between staff and patients (Stewart et al 2009) some of which can be difficult to repair (Clearly et al 2012b).

In the United Kingdom (UK), restrictive interventions began to attract significant attention following deaths that occurred during their use (Aiken et al 2011) and particularly in light of a well reported scandal at a hospital which was exposed in 2011. The government response to this (Department of Health 2012) resulted in the production of guidelines referred to as Positive and Proactive (Department of Health 2014) promoting initiatives and values to minimise the use of restrictive interventions.

A number of restraint minimisation programmes have been developed over the past 15 to 20 years to address these issues with varying degrees of success. These include 'Safewards' originating in the UK (Bowers et al 2015), and 'No Force First' (Ashcraft \& Ashcraft 2008 and the 'Engagement Model' (Borckardt et al. 2011) both originating in America. The 'Six Core Strategies' for minimising seclusion and restraint is a multilevel complex intervention targeting both organisational and individual factors in decision-making about care in acute mental health services (LeBel et al 2014). The underpinning training curriculum to reduce and prevent the use of seclusion and restraint is a comprehensive approach for practice and culture change developed by the National Association of State Mental Health Program Directors (NASMHPD) and Huckshorn, (2004; 2006; Huckshorn \& LeBel, 2009; NASMHPD, 2011). The core strategies are leadership toward organisational change; the use of data to inform practice; workforce development; person-centered tools; service user roles within inpatient settings; and debriefing techniques. The implementation of programmes informed by the Six Core Strategies approach has been associated with reduced seclusion and restraint in a number of North American evaluations (Barton et al., 2009; LeBel, 2011; Lewis et al 2009; Wieman et al., 2014, Riahi et al., 2016, Goulet et al 2017). There is also evidence of broader improvements for service users and staff including greater satisfaction with care, reduced injuries, decreased medication use, shorter admission duration and reduced staff turnover and absenteeism (Barton et al., 2009; Paxton, 2009; LeBel, 2011; Sanders, 2009, National Institute of Clinical Excellence 2015, Department of Health 2014).

Beyond North America, the Six Core Strategies has been adapted for European mental health services where the treatment culture is likely to be different (Borckardt et al. 2011). Guzman-Parra et al (2016) for example, evaluated a multi-modal approach based upon the Six Core Strategies implemented on one acute mental health ward in Spain, with significant reductions in mean usage of mechanical restraint following the intervention. Putkonen et al. (2013) also evaluated a programme informed by the strategies using a cluster, Randomised 
Control Trial design in a high-security forensic setting and reported a significant reduction in coercion (seclusion, restraint or room observation) on the intervention wards.

The study reported in this paper also adapted the Six Core Strategies and evaluated the impact of its implementation at scale in UK acute mental services across seven mental health Trusts. The adaptation was based on extensive consultation with stakeholders and became known as 'REsTRAIN YOURSELF'. The core strategies were operationalized through a number of specific interventions targeted for a UK context as follows: (1) setting team goals for the reduction of restraint; (2) reflecting upon the use of restraint and personal communication styles (through reporting and analysing every restraint incident over a period of time); (3) using approaches to help patients and staff ascertain needs and challenges with regards to aggression on the ward; (4) employing partnership working strategies to reduce restraint such as 'advance directives' (my safety plan), and positive verbal and non-verbal communication; (5) exploring environmental challenges to make appropriate changes (both physical and procedural); and (6) debriefing following incidents or near misses of restraint.

\section{Insert Figure 1}

The full implementation process and project evaluation, including secondary outcomes such as the perceived impact on the ward environment and therapeutic relationships, has been more fully, reported upon elsewhere (Duxbury et al 2016, Duxbury et al 2019). The aim of the phase of the study reported here however, was to examine changes in physical restraint use on participating wards following introduction of the intervention. The main hypothesis was that restraint use would be lower on intervention wards after the introduction of REsTRAIN YOURSELF.

\section{METHODS}

Study design

The overarching evaluation consisted of a non-randomised controlled trial comprising a mixture of qualitative and quantitative approaches including the measurement of physical restraint rates, the outcome of staff and patient surveys and interviews and ethnographic data. For the purpose of this paper only the restraint data outcomes are reported upon here. Restraint rates on acute mental health care wards receiving the intervention were compared with those on control wards over 3 study phases (baseline, implementation and adoption). This design is commonly used in studies involving complex interventions in health services, which are described as interventions that contain several interacting components (Criag et al 2008). Other characteristics that evaluators should take into account include a good 
theoretical understanding of what is needed and how the intervention causes change; The exploration of any lack of effect which may reflect implementation failure (or teething problems) rather than genuine ineffectiveness. A thorough process evaluation to identify implementation problems; The consideration of a range of measures so that any unintended consequences are picked up where possible; And finally, information outlining the adaptation of the intervention to local settings.

Whilst many issues surrounding evaluation of complex interventions are still debated, guidelines are there to help researchers, funders, and other decision makers to make appropriate methodological and practical choices (Craig et al 2008).

Fidelity in studies of this sort is also an important issue, however it is not straightforward in relation to complex interventions (Hawe et al 2004). Whilst in some evaluations, such as those seeking to identify active ingredients within a complex intervention, strict standardisation may be required and controls put in place to limit variation in implementation (Farmer et al 2007), others are designed to be adapted to local circumstances. In this study, the six core strategies were applied as part of the implementation of RESTRAIN YOURSELF, however, some local nuances were catered for dependent upon local need. For example, targeted training in the prevention and management of self-harm and for those with specific disorders such as personality disorders, was provided to some wards where this was identified as a significant contributory factor to the development of conflict. The key is to be clear about how much change or adaptation is permissible and to record variations in implementation so that fidelity can be assessed in relation to the degree of standardisation required by the study protocol.

\section{Setting and participants}

The study took place on 14 acute, adult wards across various sites in the North West of England comprising five counties; Cheshire, Greater Manchester, Merseyside, Lancashire and Cumbria with over 7 million residents. In 2012, the region's physical restraint rates were reported to be high when compared to the average for England at that time (MIND 2013). For example the seven Trusts averaged 1221 restraint epiosdes in 2012 compared with a national average of 455 . The average number of patients restrained, use of 'face down' restraint, restraint-related injuries and restraint-related complaints in these Trusts were all significantly greater than the national average (MIND 2013). The number of patients restrained was 7073 (mean 884, range 62-1965, national average 247). The four Trusts that reported face down data recorded 271 face down restraints (mean 68, range 02-175, 
national average 65), whilst all the Trusts that provided restraint-related injury data recorded 552 restraint related injuries (mean 79, range 0-200, national average 7 ). There were zero restraint related deaths and 26 restraint-related complaints across all seven Trusts (mean 3 , range $0-8$, national average 2 ).

Two acute care wards were targeted from all eligible acute wards within each participating organization in negotiation with each Trust. The research team then endeavoured to allocate matched wards for each Trust taking into account restraint use, number of beds and patient demographics. This information was then fed back to the Trust leads for the project. Whilst allocations were then made and matched, some Trusts communicated that they were limited in the wards they could use due to competing interventions that had been introduced in some areas. Therefore, agreements between the research team and the Trusts had to reached meaning that in some instances, non-matched samples had to be used. Whilst the research team initially endeavoured to use matched criteria e.g. restraint rates, acuity, gender mix, when selecting a comparison ward in the same Trust, this process was hampered by factors external to the study. For example, the simultaneous implementation of other conflict minimisation interventions, led to the exclusion of what might have been well-matched wards resulting in a reduced pool of wards from which to select participants. Therefore, there were some differences between ward pairs at baseline. The intervention wards (total $n=144$ beds, mean $=20.1$ beds per ward) and control wards (total $n=147$ beds, mean $=21.0$ beds per ward) were primarily mixed gender but included single sex wards also ( 2 female-only and 1 male-only in each group). All wards offered a combination of pharmacological and psychosocial interventions over relatively short admission durations (circa 15 days).

Intervention

Over the period of the project, and within all the participating Trusts, a range of different innovations were rolled out on the implementation wards within a six core strategy framework.

Insert table 1

As part of our team, a dedicated improvement adviser worked on the wards one day a week to support the implementation of the approach using a number of strategies including:

- The identification of potential change ideas with staff in line with REsTRAIN Yourself. 
- The exploration of Ideas and changes that the individual teams would test using Plan, Do, See, Act cycles within their selected ward.

- The identification of group and individual roles and ownership whereby each member of the team commits to individual actions in order to achieve agreed goals.

To prepare the participating ward teams a 'Train the Trainer' model was used to roll out training. An online toolkit, which incorporates theory, Quality Improvement methodological techniques, case examples and top tips, was developed to support both the 2 day face to face training events and ongoing implementation of the approach.

(https://www.aquanw.nhs.uk/resources/restrain-yourself-toolkit/20917). Local steering groups were set up to progress ongoing spread and sustainability. The approach focused on identified champions for each ward and attendance at action learning sessions on a monthly basis.

When using a complex intervention of this type, without a complete published description of interventions, clinicians and patients cannot reliably implement approaches that are shown to be useful, and other researchers cannot replicate or build on research findings. To assist with future work in this area and to aid implementation and replication, the Template for Intervention Description and Replication (TIDieR) checklist and guide is attached as an appendix to this paper. This may improve the reporting of using the RESTRAIN YOURSELF intervention and make it easier to use the information (Hoffman et al 2014).

\section{Procedure}

There were three study phases during the course of the project. These were baseline, implementation and adoption. The implementation phase covered the period when the RESTRAIN YOURSELF adviser was active on the ward (duration mean per ward $=5$ months, range $=3.5-5.5$ months). The baseline phase (mean duration $=13.6$ months, range $=8.1-$ 18.3 months) covered the study period prior to this implementation activity and the adoption phase (mean duration $=7.9$ months, range $=2.4-13.1$ months) covered the period after the Improvement Advisor stopped visiting the ward. In this phase, staff were encouraged to carry on REsTRAIN YOURSELF implementation without active external support from the project and the continued use of their local 'champions'. The baseline and adoption phases covered at least 6 months each. In order to allocate the wards to a study group, a project lead from each Trust produced a table of all their inpatient wards including their characteristics and any similar initiatives that were being introduced on each ward such as 'Safewards'. 
During the baseline phase care was delivered following standard protocols in force at the participating trust at the time. The variation in baseline duration occurred because data were available from all trusts at the start of the study but the intervention was implemented in waves at three different time points. Therefore early implementers had a shorter baseline than later implementers. Restraint rates were monitored throughout the baseline period and not at a single time point. The duration of the final adoption phase varied between wards. Whilst this has been controlled for statistically, it is not possible to estimate how long any changes persisted across periods within the adoption phase or indeed beyond. The total study duration was 16.7 months on all wards. Wards varied only in terms of what proportion of this overall time period involved each study phase as outlined.

\section{Analysis}

The primary outcome of interest was the number of physical restraint events logged on the organization's adverse incidents recording system during the study period (January 2015February 2016). Physical restraint was defined at the time of the study as "a skilled hands-on method involving trained, designated healthcare professionals" designed to safely immobilise an individual to prevent them from harming themselves, endangering others or seriously compromising the therapeutic environment (National Institute of Clinical Excellence, 2015). It can be implemented with the service user on the floor or in a standing or seated position. Anonymised prospective case-level data from incident records was provided for every episode of physical restraint occurring on the intervention and comparator wards during the specified period.

Restraint event rates per 1000 bed-days with 95\% confidence intervals were calculated for the intervention and comparator wards across the study period. Associations between exposure to the intervention and restraint frequencies were tested using chi-squared analysis. Data analyses were conducted at the cluster level only and no individual level data analyses were performed.

\section{Ethics}

The study was approved by the National Research Ethics Service of the Health Research Authority for England (ref. 14/YH/0164) in July 2014. Consent for access to anonymised secondary data was not required by the research ethics committee and was not obtained. 


\section{RESULTS}

Restraint rates

In total, 1680 physical restraint incidents were logged over the full study period (range across organisations $=68-492$ incidents). This equates to 0.15 restraints per day or approximately 1 restraint every 6.5 days. Overall, there was an average reduction of physical restraint rates across the intervention wards of $22 \%$. More restraint took place overall on REsTRAIN YOURSELF intervention wards ( $\mathrm{n}=980,58.3 \%$ of all restraint incidents) than comparator wards ( $n=700,41.6 \%$ ). The restraint rate was significantly lower on the intervention wards in the adoption phase (6.62 events/1000 bed-days, $95 \% \mathrm{Cl} 5.53-7.72)$ compared to the baseline phase $(9.38,95 \% \mathrm{Cl} 8.19-10.55)$ (see Figure 2). However there was a substantial temporary increase on the intervention wards during the implementation phase $(10.76,95 \%$ $\mathrm{Cl}$ 9.34-12.19) prior to the decrease in the adoption phase. Also there were substantial variations in trends across the intervention wards with decreases exceeding $18 \%$ on five wards and increases exceeding $20 \%$ on two wards (see Table 2). There were no significant changes in restraint rates on the comparator wards over the same period (baseline: 5.33, 95\% Cl 4.45-6.20; implementation: 5.65, 95\% Cl 4.62-6.67; adoption: 7.22, 95\% Cl 6.018.42). The intervention wards had significantly higher restraint rates at baseline than the comparator wards but the rates were comparable in the final adoption phase of the study.

The association between ward type (comparator versus implementation) and study phase was statistically significant for this analysis (chi-squared $=39.15$, df $=13 p<$. 0002; effect size $=62 \%$ reduction relative to comparator wards).

Insert Table 2 and Figure 2

\section{DISCUSSION}

This large-scale study sought to evaluate the implementation of a restraint minimisation programme underpinned by principles of prevention and trauma informed care, in a number of UK acute mental health settings. However, only the impact on physical restraint is reported upon in this paper. There is preliminary evidence here of some potential association between the implementation of RESTRAIN YOURSELF and reduced use of restraint not dissimilar to that reported in other studies where complex multidimensional approaches based upon the Six Core Strategies have been used (Riahi et al 2016, Putkonen et al 2013, Hernandez et al 2017). Caution is required however, when assessing the level of association between implementation and outcomes given the limitations reported. Notable 
concerns include a lack of randomisation or standardized implementation, the use of nonmatched wards and limited feasibility work.

With regard to restraint rates, the most stringent test involves comparing the adoption phase when staff were expected to sustain the changes in practice without active support from the adviser, with the baseline levels prior to implementation. The use of restraint was significantly lower in this latter phase on wards where the Intervention took place. This was achieved in the context of increased use of restraint in comparable settings over the same period. This suggests that the intervention could have been successfully embedded into the participating ward cultures, potentially having some effect on reducing staff reliance on coercive measures. Riahi et al (2016) reported similar results in reducing seclusion and mechanical restraint when approaches such as the Six Core Strategies promote enhanced staff knowledge, skills and attitudes that results in organisational changes to cultures. This is also true in the reduction of conflict and containment as evidenced in the use of Safewards (Bowers et al 2015). Hernandez et al (2017) found a step decrease in seclusion hours and restraint over time using an interrupted time serious analysis pre and post implementation of a multidimensional approach to restraint minimization based upon the Six Core Strategies. They concluded that leadership and daily rounds were particularly valuable in reducing average restraint and seclusion hours.

The significant reduction in restraint rates between the two phases is in line with that reported by Putkonen et al. (2013) in their Randomised Control Trial in a high secure forensic setting in Finland. They found a scale of reduction (29\% in rates per 100 beds/observation days) to be substantial exceeding that reported by Blair et al. (2017) and Lewis et al. (2009) in their respective studies. Statistically significant associations were also found between the intervention and a decrease in both the number of seclusions $(p<0.01)$ and the duration of seclusion per admission $(p<0.001)$. These preliminary results support the conclusion that this intervention was effective in reducing the use of seclusion.

In our project the non-randomised nature of the design inevitably limits the degree to which this effect can be attributed to the RESTRAIN YOURSELF programme alone. Whilst the initial intention was to match wards on a number of variables, operational factors such as the limited pool of available wards ultimately made this difficult to achieve. In particular, restraint rates were higher on the implementation wards than the comparator wards at baseline. This may suggest some underlying differences between the wards in each group, which limit the direct conclusions that can be drawn about any causal effect of the intervention. It should also be noted that the base rates of restraint were low on some of these wards and the trend 
varied both within and between wards. This indicates some caution in making any interpretations of the relationship between the intervention and restraint rates.

The non-significant increase during the implementation phase is also noteworthy indicating either challenges in adjusting to the new approach or a reduced threshold amongst staff for the reporting of incidents due to the highlighting of the issue through participation in the study. Improved reporting systems as part of the intervention strategy may also have increased sensitization to recording events during the early phases. Bowers et al (2015) found a similar rise initially when implementing Safewards.

The variation between wards is also worth noting. The combined trend across all the wards for a reduction in restraint masks different patterns on individual wards. Whilst all but one of the wards were similar in that they reported a decrease following implementation, these reductions varied from $65 \%$ to $8 \%$. Given the complexity of the intervention and the context, there are numerous potential factors, which may have influenced the degree of reduction including reporting differences and programme fidelity but unfortunately, it is not possible to examine these factors in the current design. Future research testing this intervention could include additional measures to consider these potential factors and greater feasibility work. It is of concern also that one ward reported an increase in restraint after implementation of the programme. Again, it is not possible to test any speculation about possible causes for this counter-intuitive trend but it may reflect improved reporting as one element of the intervention and/or difficulties we encountered engaging with this ward team in comparison to others.

From the evaluation data reported more fully elsewhere (Duxbury et al 2016), we were able to identify that arguably, across all of the wards in the study, both before and after the implementation of RESTRAIN YOURSELF, embedded cultures and practices shared a number of common characteristics. This was so despite a complex mix of different environments, staff and service user views and behaviour. All of this took place against a backdrop of constraining structural and resource issues, including managerial reorganisations and service relocations in some of the Trusts. Further qualitative findings reporting upon the impact of processes aligned to this project can be found in Duxbury et al (2019). Challenges and positive effects are outlined from a nursing perspective many of which are not unique to mental health settings (Bevan \& Fairman 2014, Grol et al 2013, Robert et al 2015), other mental health care change initiatives (Brennan et al. 2006, Woltmann et al. 2008, Chambers et al 1998, McAndrew et al 2014) or conflict minimisation projects (Bowers et al 2006, Riahi et al 2016). 
Despite our success in reducing the use of restraint over the study period, the pull to coercion is powerful and subject to social forces beyond the influence of individual nurses and teams; being framed by a significant governance attachment to risk management within a wider frame of a 'risk society'. Staff have to gain faith in the instrumental value of alternative approaches to volatile and distressed individuals such as REsTRAIN YOURSELF, or in the context of self-harm. Pettit et al (2017) reported that where there is no access to seclusion, staff as a team are slower to initiate restraint, suggesting an achievable positive service shift. Staff have to believe however, that changing practices which are managerially supported are indeed, legitimated. Despite some counter-veiling forces such as staff sickness and resistance, the intervention may have made some progressive inroads into reducing instances of restraint and altering various ward practices. Staff were very positive about the approach overall and found the results to be both convincing and reassuring (Duxbury et al 2019).

\section{LIMITATIONS}

Whilst this study has shown promising results, a number of limitations are evident as highlighted in the discussion section. Firstly, despite our initial best efforts, the wards were not sufficiently matched due to pressures on the wards and endeavours to avoid contamination and competing initiatives. Secondly, a number of confounding variables could have influenced the reduction of the restraint figures and not solely the complex intervention in its entirety. For example, we were unable to report upon a number of issues which may have been influential including the number of patients treated in the respective periods of time, outcomes other than number of restraint episodes per time and bed or on the frequency of violent incidents over the respective periods.

The outcome examined here, restraint events, is a robust measure but inevitably restraint events may be influenced by other contextual factors such as admission rates and general level of violence. The unavailability of data on these other factors due to project resource constraints prevented us from conducting a more complex analysis which may have identified additional interacting variables in a broader restraint prediction model. Future research in this area should aim to capture this wider picture.

Finally, whilst we know that the intervention has continued in a number of Trusts anecdotally and that teams report its continued success, we do not have any data that looked at the fidelity or the sustainability of the intervention. That said the implementation toolkit is freely available online and breaks down all the stages of the RESTRAIN YOURSELF approach so 
that the process can be replicated (https://www.aquanw.nhs.uk/resources/restrainyourself-toolkit/20917).

\section{CONCLUSION}

Acknowledging the limitations of a non-randomised evaluation and the non standardisation of some aspects of the implementation process outlined above, we were able to report an average reduction of restraint by $22 \%$ on the implementation wards over a six-month period. Some wards recorded significantly greater figures than this. However, whether this can be soley attributed to RESTRAIN YOURSELF is yet to be established. Our findings did demonstrate that reductions in the use of restraint and changes to the psychosocial ward environment are potentially achievable with the support of multidimensional organisational models (Hernandez et al 2017, Putkonen et al 2013, Goulet et al 2017). Interventions such as REsTRAIN YOURSELF, which enable teams to take collective ownership of changes in their own ward environments, and to build positive engagement with service users through data informed practice and person-centred tools, are an important step in the right direction. This promotes the recognition of service users' existing trauma, as opposed to relying upon readily available coercive measures (Hernandez et al 2017, Blair et al 2017). With additional investment in overall resources and managerial support to minimise the use of practices such as restraint, as advocated by Huckshorn (2004) and as seen in the work of Riahi et al (2016), even better outcomes might be possible, The use of feasibility studies to explore and evidence usable and acceptable approaches to implement the Six Core Strategies in different geographical and practice settings in the future would be welcomed.

\section{RELEVANCE FOR CLINICAL PRACTICE}

Organisational models based upon approaches such as the six core strategies are increasingly proving to be popular in reducing restrictive interventions such as physical restraint. Given the current trend to minimize restrictive interventions globally and the underpinning political and policy agendas, this is an opportunity for teams to use evidence based, multimodal preventative, trauma informed and person-centered tools, to both reduce conflict on inpatient wards but also to minimise practices which are increasingly seen to be physically and psychologically traumatising to service users and staff. 


\section{REFERENCES}

Agenda (2017) $A$ briefing document on the use of restraint against women and girls March 2017 Agenda: London

Aguilera-Serrano, C., Guzman-Parra, J., Garcia-Sanchez, J.A., Moreno-Küstner, B. and Mayoral-Cleries, F. (2018) Variables Associated with the Subjective Experience of Coercive Measures in Psychiatric Inpatients: A Systematic Review. The Canadian Journal of Psychiatry, 63(2), 129-144.

Ashcraft L, Anthony WA, Jaccard S. (2008) Rein in seclusion and restraints: they are not compatible with recovery-oriented services. Behav Healthc. 1;28 (12):6-8.

Aiken F, Duxbury J, Dale C \& Harbison I (2011) Report on the Theories on Restraint Related Deaths in the UK, Ministry of Justice, London

Barton, SA, Johnson MR \& Price LV (2009) Achieving restraint-free on an inpatient behavioral health unit. Journal of Psychosocial Nursing and Mental Health Service, 47(1), 34-40.

Bevan, H \& Fairman, S (2014) The new era of thinking and practice in change transformation: A Call to Action for Leaders of Health and Care, NHSIQ (NHS Improving Quality). http://media.nhsiq.nhs.uk/whitepaper/html5/index.html?page=1

Blair EW, Woolley S, Szarek BL et al (2017) Reduction of Seclusion and Restraint in an Inpatient Psychiatric Setting: A Pilot Study. Psychiatr Q. 2017 Mar;88(1):1-7

Bonner G, Lowe T, Rawcliffe D, Wellman N. (2002) Trauma for all: a pilot study of the subjective experience of physical restraint for mental health inpatients and staff in the UK. $\underline{J}$ Psychiatr Ment Health Nurs. 1;9(4):465-73.

Borckardt, J.J., Madan, A., Grubaugh, A.L., Danielson, C.K., Pelic, C.G., Hardesty, S.J., Hanson, R., Herbert, J., Cooney, H., Benson, A. and Frueh, B.C. (2011) Systematic investigation of initiatives to reduce seclusion and restraint in a state psychiatric hospital. Psychiatric Services, 62(5), 477-483.

Bowers L, James K, Quirk A, Simpson A, Stewart D, Hodsoll J. (2015) Reducing conflict and containment rates on acute psychiatric wards: The Safewards cluster randomised controlled trial. Int J Nurs Stud. 2015; 52(9):1412-22.

Bowers L, Alexander J, Simpson A, Ryan C \& Carr-Walker P (2004) Cultures of psychiatry and the professional socialization process: the case of containment methods for disturbed patients. Nurse Education Today, 24(6), 435-442.

Bowers L, Douzenis A, Galeazzi G, Forghieri M, Tsopelas C, Simpson A \& Allan T (2005) Disruptive and dangerous behaviour by patients on acute psychiatric wards in three European centres. Social Psychiatry and Psychiatric Epidemiology, 40(10), 822-828.

Bowers, L., Nijman, H., Allan, T., Simpson, A., Warren, J. \& Turner, L. (2006) Prevention and management of aggression training and violent incidents on UK acute psychiatric wards. Psychiatric Services, 57(7), 1022-1026. 
Bowers L, van der Werf, Vokkolainen A, Muir Cochrane E, Allan T \& Alexander J (2007) International variation in containment measures for disturbed psychiatric inpatients: A comparative questionnaire survey, International Journal of Nursing Studies, 44, 357 - 364.

Bowers L (2014) Safewards: a new model of conflict and containment on psychiatric wards Journal of Psychiatric and Mental Health Nursing, 21, 499-508

Brennan, G., Flood, C. \& Bowers, L. (2006) Constraints and blocks to change and improvement on acute psychiatric wards-lessons from the City Nurses project. Journal of psychiatric and mental health nursing, 13(5), 475-482.

Chambers, M. (1998) Interpersonal mental health nursing: Research issues and challenges. Journal of Psychiatric and Mental Health Nursing, 5, 203-211.

Cleary M. (1999) 'Something always comes up': nurse-patient interaction in an acute psychiatric setting. Journal of Psychiatric and Mental Health Nursing, 6(6), 469-477.

Cleary, M., Horsfall, J., O'Hara-Aarons, M., Jackson, D. and Hunt, G.E. (2012a) Mental health nurses' perceptions of good work in an acute setting. International Journal of Mental Health Nursing, 21(5), 471-479.

Cleary, M., Hunt, G.E., Horsfall, J. and Deacon, M. (2012b) Nurse-patient interaction in acute adult inpatient mental health units: A review and synthesis of qualitative studies. Issues in Mental Health Nursing, 33(2), 66-79.

Cowman, S., Björkdahl, A., Clarke, E., Gethin, G. and Maguire, J. (2017) A descriptive survey study of violence management and priorities among psychiatric staff in mental health services, across seventeen European countries. BMC health services research, 17(1), p.59.

Dack C, Ross J \& Bowers L (2012) The relationship between attitudes towards different containment measures and their usage in a national sample of psychiatric inpatients. Journal of Psychiatric and Mental Health Nursing, 19(7), 577-586.

Department of Health. (2012) Transforming care: A national response to Winterbourne View Hospital Department of Health Review: Final Report. Department of Health

Department of Health.(2014) Positive and Proactive Care: reducing the need for restrictive interventions. Department of Health

Duxbury J (2002) An evaluation of staff and patient views of and strategies employed to manage inpatient aggression and violence on one mental health unit: a pluralistic design. Journal of Psychiatric and Mental Health Nursing, 9, 325-337.

Duxbury J (2003) Testing a new tool: The Management of Aggression and Violence Scale (MAVAS). Nurse Researcher, 62(5), 596-606.

Duxbury J \& Whittington R (2005) Causes and management of patient aggression and violence: staff and patient perspective. Journal of Advanced Nursing, 50, 469-478.

Duxbury J, Aiken F \& Dale C (2011) Deaths in custody: the role of restraint. Journal of Learning Disabilities and Offending Behaviour, 2(4), 178 - 189. 
Duxbury JA (2015) Editorial: Minimizing the use of coercive practices in mental health: the perfect storm, Journal of Psychiatric and Mental Health Nursing, 22, 89-91

Duxbury J (2016) Implementing the Six Core Strategies (RESTRAIN YOURSELF): Working in partnership to minimise harm to patients and staff by reducing the use of physical restraint. Health Foundation Report: London

Duxbury J, Thomson G, Scholes A, Jones F, Baker J, Downe S, Greenwood, P, Price O, Whittington $R$ and McKeown M (2019). Staff experiences and understandings of the REsTRAIN Yourself initiative to minimize the use of physical restraint on mental health wards International Journal of Mental Health Nursing doi: 10.1111/inm.12577

Farmer A, Wade A, Goyder E, Yudkin P, French D, Craven A, et al. (2007) Impact of selfmonitoring of blood glucose in the management of patients with non-insulin treated diabetes: open parallel group randomised trial. BMJ;335:132-9.

Flood C, Bowers L, Parkin D. (2008) Estimating the costs of conflict and containment on adult acute inpatient psychiatric wards. Nurs Econ;26 (5):325.

Gilburt, H., Rose, D. \& Slade, M. (2008) The importance of relationships in mental health care: A qualitative study of service users' experiences of psychiatric hospital admission in the UK. BMC Health Services Research, 8(1), 1.

Glover RW (2005) Special section on seclusion and restraint: Commentary Reducing the use Of seclusion and restraint: A NASMHPD priority. Psychiatric Services, 56(9), 1141-1142.

Goulet, M.H., Larue, C. and Dumais, A., (2017). Evaluation of seclusion and restraint reduction programs in mental health: A systematic review. Aggression and violent behavior, 34, pp.139-146

Grol, R., Wensing, M., Eccles, M. \& Davis, D. (eds.) (2013) Improving patient care: the implementation of change in health care. Oxford, John Wiley \& Sons.

Guzman-Parra, J., Aguilera Serrano, C., García-Sánchez, J.A., Pino-Benítez, I., Alba-Vallejo, M., Moreno-Küstner, B. and Mayoral-Cleries, F. (2016) Effectiveness of a multimodal intervention program for restraint prevention in an acute Spanish psychiatric ward. Journal of the American Psychiatric Nurses Association, 22(3), 233-241.

Hawe P, Shiell A, Riley T. (2004) Complex interventions: how "out of control" can a randomised trial be? BMJ;328:1561-3.

Hernandez A, Riahi S, Stuckey MI et al (2017) Multidimensional approach to restraint minimization: The journey of a specialized mental health organization International Journal of Mental Health Nursing, Volume 26, Issue 5, Pages 482-490

Hoffman TC, Glasziou PP, Boutron I et al (2014) Better reporting of interventions: template for intervention description and replication

(TIDieR) checklist and guide, BMJ, 348:g1687 doi: 10.1136/bmj.g1687,pp. 1-12

Huckshorn K (2004) Reducing seclusion restraint in mental health use settings: core strategies for prevention. Journal of Psychosocial Nursing and Mental Health Services, 42, 22-33. 
Huckshorn K (2006) Re-Designing State Mental Health Policy to Prevent the Use of Seclusion and Restraint. Administration and Policy in Mental Health and Mental Health Services Research, 33, 482-491.

Huckshorn KA (2004) Reducing the use of seclusion and restraint in mental health systems: A public health prevention approach with interventions. Journal of Psychosocial Nursing and Mental Health Services, 42 (9): 22-33.

Huckshorn KA (2006) Re-designing state mental health policy to prevent the use of seclusion and restraint. Administration and Policy in Mental Health, 33(4), 482-491.

Huckshorn KA \& LeBel JL (2009) Improving safety in mental health treatment settings: Preventing conflict, violence and the use of seclusion and restraint. In SS Sharfstein, FB

Laker C, Gray R, Flach C. (2010) Case study evaluating the impact of de-escalation and physical intervention training. J Psychiatr Ment Health Nurs;17(3):222-8.

Lebel J, Duxbury J, Putkonen A et al (2014) Multinational Experiences in Reducing and Preventing the Use of Restraint and Seclusion Journal of Psychosocial Nursing and Mental Health Services, 52 (11). pp. 22-29

LeBel J (2011) The Business Case for Preventing and Reducing Restraint and Seclusion Use. HHS Publication No. (SMA) 11-4632. Rockville, MD: Center for Mental Health Services, Substance Abuse and Mental Health Services Administration.

LeBel J \& Goldstein R (2005) The economic cost of using restraint and the value added by restraint reduction or elimination. Psychiatric Services, 56(9), 1109-14.

Lee S, Wright S, Sayer J, Parr A-M, Gray R, Gournay K. (2009) Physical restraint training for nurses in English and Welsh psychiatric intensive care and regional secure units. $\underline{\mathrm{J} \text { Ment }}$ Health.

Lewis M, Taylor K \& Parks J (2009). Crisis prevention management: A program to reduce the use of seclusion and restraint in an inpatient mental health setting. Issues in Mental Health Nursing, 30(3),159-164.

Livingston JD, Verdun-Jones S, Brink J, Lussier P, Nicholls T. (2010) A narrative review of the effectiveness of aggression management training programs for psychiatric hospital staff. J Forensic Nurs. 1;6 (1):15-28.

Luciano, M., Sampogna, G., Del Vecchio, V., Pingani, L., Palumbo, C., De Rosa, C., Catapano, F. and Fiorillo, A. (2014) Use of coercive measures in mental health practice and its impact on outcome: a critical review. Expert review of neurotherapeutics, 14(2), 131-141.

McAndrew, S., Chambers, M., Nolan, F., Thomas, B. and Watts, P. (2014) Measuring the evidence: Reviewing the literature of the measurement of therapeutic engagement in acute mental health inpatient wards. International journal of mental health nursing, 23(3), 212-220.

MIND (2013) Mental health crisis care: physical restraint in crisis: A report on physical restraint in hospital settings in England. MIND: London.

Mohr, W.K., Petti, T.A. and Mohr, B.D.(2003). Adverse effects associated with physical restraint. The Canadian Journal of Psychiatry, 48(5), pp.330-337. 
Muralidharan S, Fenton M. 2006 Containment strategies for people with serious mental illness. Cochrane Database Syst Rev; 3.

National Association of State Mental Health Program Directors (NASMHPD) (2011) National executive training institute: A training curriculum for the reduction of seclusion and restraint, 9th Edition. Alexandria, VA. National Association of State Mental Health Program Directors, Office of Technical Assistance.

National Collaborating Centre for Mental Health. (2015) Violence and Aggression Short-term management in mental health, health and community settings Updated edition NICE Guideline NG10.

National Institute of Clinical Excellence (2005) Violence; The Short Term Management of Disturbed/Violent Behaviour in Psychiatric In-Patient Settings and Emergency Departments. NICE, London.

Noorthoorn E, Lepping P, Janssen W, Hoogendoorn A, Nijman H, Widdershoven G, et al. (2015) One-year incidence and prevalence of seclusion: Dutch findings in an international perspective. Soc Psychiatry Psychiatr Epidemiol. 50(12):1857-69.

Paterson B, Bradley P, Stark C, Saddler D, Leadbetter D, Allen D. (2003) Deaths associated with restraint use in health and social care in the UK. The results of a preliminary survey. Psychiatr Ment Health Nurs. 10 (1):3-15.

Pettit SA, Bowers L, Tulloch A, Alexis E. et al (2016) Acceptability and use of coercive methods across differing service configurations with and without seclusion and/or psychiatric intensive care units Journal of Advanced Nursing 73(4), 966-976.

Putkonen A, Kuivalainen S, Louheranta O, Repo-Tiihonen E, Ryynänen O-P, Kautiainen H, et al. (2013) Cluster-Randomized Controlled Trial of Reducing Seclusion and Restraint in Secured Care of Men With Schizophrenia. Psychiatr Serv; 64(9):850-5.

Riahi, S., Thomson, G. and Duxbury, J. (2016) An integrative review exploring decision-making factors influencing mental health nurses in the use of restraint. Journal of Psychiatric and Mental Health Nursing, 23(2), 116-128.

Robert, G., Cornwell, J., Locock, L., Purushotham, A., Sturmey, G. \& Gager, M. (2015) Patients and staff as codesigners of healthcare services. BMJ, 350, 7714.

Rose, D., Evans, J., Laker, C. \& Wykes, T. (2015) Life in acute mental health settings: experiences and perceptions of service users and nurses. Epidemiology and Psychiatric Sciences, 24, 90-96.

Sanders K (2009) The effects of an action plan, staff training, management support and Monitoring on restraint use and costs of work-related injuries. Journal of Applied Research in Intellectual Disabilities, 22(2), 216-220.

Soininen, P., Kontio, R., Joffe, G. and Putkonen, H. (2016) Patient Experience of Coercive Measures. In The Use of Coercive Measures in Forensic Psychiatric Care (pp. 255-270). Springer, Cham.

Steinert T, Eisele F, Goeser U, Tschoeke S, Uhlmann C, Schmid P. (2008) Successful interventions on an organisational level to reduce violence and coercive interventions in in- 
patients with adjustment disorders and personality disorders. $\underline{\text { Clin Pract Epidemiol Ment }}$ Health;4(1):27.

Steinert T, Lepping P, Bernhardsgrütter R, Conca A, Hatling T, Janssen W, et al. (2010) Incidence of seclusion and restraint in psychiatric hospitals: a literature review and survey of international trends. Soc Psychiatry Psychiatr Epidemiol. 45(9):889-97.

Stewart D, Bowers L, Simpson A, Ryan C, Tziggili M. (2009) Manual restraint of adult psychiatric inpatients: a literature review. J Psychiatr Ment Health Nurs;16 (8):749-57.

Sweeney, A., Clement, S., Filson, B. and Kennedy, A., (2016). Trauma-informed mental healthcare in the UK: what is it and how can we further its development?. Mental Health Review Journal, 21(3), pp.174-192.

Tunde Ayinmode M \& Little J (2004) Use of Seclusion in a psychiatric acute inpatient unit. Australasian Psychiatry, 12 (4), 347-351.

Wieman DA, Camacho-Gonsalves T, Huckshorn K \& Leff S (2014) Multisite Study of an Evidence-Based Practice to Reduce Seclusion and Restraint in Psychiatric Inpatient Facilities PSYCHIATRIC SERVICES, Vol. 65 No. 3

Woltmann EM, Whitley R, McHugo GJ, Brunette M, Torrey WC, Coots L, Lynde D, Drake RE. (2008) The role of staff turnover in the implementation of evidence-based practices in mental health care. Psychiatr Serv. Jul;59(7):732-7. doi: 10.1176/appi.ps.59.7.732.

(https://www.aquanw.nhs.uk/resources/restrain-yourself-toolkit/20917). 